\title{
Methylcitrate cycle activation during adaptation of Fusarium solani and Fusarium verticillioides to propionyl-CoA-generating carbon sources
}

\author{
Correspondence \\ Matthias Brock \\ Matthias.Brock@hki-jena.de
}

Received 16 June 2009

Revised 20 July 2009

Accepted 31 July 2009

\section{Nicole Domin, ${ }^{1}$ Duncan Wilson ${ }^{2}$ and Matthias Brock ${ }^{1}$}

\author{
${ }^{1}$ Microbial Biochemistry and Physiology, Leibniz Institute for Natural Product Research and \\ Infection Biology, Hans Knoell Institute, Beutenbergstr. 11a, 07745 Jena, Germany \\ ${ }^{2}$ Microbial Pathogenicity Mechanisms, Leibniz Institute for Natural Product Research and Infection \\ Biology, Hans Knoell Institute, Beutenbergstr. 11a, 07745 Jena, Germany
}

\section{INTRODUCTION}

Most, if not all, aerobic micro-organisms have to cope with the formation of propionyl-CoA. This metabolite can be formed from the direct activation of propionate (a common carbon source found in soil), or from the degradation of either odd-chain fatty acids or amino acids such as isoleucine, valine and methionine (Brock et al., 2000; Voet \& Voet, 2004; Maerker et al., 2005). Accumulation of propionyl-CoA exerts toxic effects by inhibiting several enzymes of primary metabolism, such as the pyruvate dehydrogenase complex, succinyl-CoA synthase and ATP:citrate lyase (Brock \& Buckel, 2004). To avoid this accumulation, several bacteria and mammals utilize the so-called methylmalonyl-CoA pathway for converting propionyl-CoA into the citric acid cycle intermediate succinyl-CoA (Martens et al., 2002). However, the key enzyme of this pathway, the coenzyme $\mathrm{B}_{12}$-dependent methylmalonyl-CoA mutase, is absent in fungi (Ledley et al., 1991). Most fungi instead use the coenzyme $\mathrm{B}_{12}$-independent methylcitrate cycle for the efficient degradation of propionyl-CoA. This pathway is also present in several bacteria and has been studied mainly in the bacteria Escherichia coli (Brock et al., 2002; Textor et al., 1997) and Salmonella typhimurium (Horswill \& Escalante-Semerena, 2001) and the fungi Yarrowia lipolytica (Uchiyama et al., 1982), Saccharomyces cerevisiae (Luttik et al., 2000), Aspergillus nidulans (Brock et al., 2000, 2001; Brock \& Buckel, 2004; Brock, 2005; Zhang et al., 2004) and Aspergillus fumigatus (Ibrahim-Granet et al., 2008; Maerker et al., 2005). The methylcitrate pathway is characterized by the initial condensation of propionyl-CoA with oxaloacetate via methylcitrate synthase, forming the first cycle-specific intermediate, methylcitrate. Methylcitrate undergoes an isomerization event, forming methylisocitrate, which is finally cleaved into pyruvate and succinate by a cycle-specific methylisocitrate lyase. This cleavage completes the $\alpha$-oxidation of propionate to pyruvate, which can then be used for energy and biomass formation by standard enzymes of primary metabolism (Brock et al., 2000). 
Deletion of the methylcitrate synthase gene from $A$. nidulans and A. fumigatus leads to an accumulation of propionyl-CoA in the presence of propionyl-CoA-generating carbon sources and during starvation. Interestingly, the accumulation of propionyl-CoA not only reduces the growth rate of these fungi due to the inhibition of primary metabolism under in vitro conditions, as outlined above (Brock \& Buckel, 2004), it also leads to the attenuation in virulence of $A$. fumigatus in a wax moth larvae model (Maerker et al., 2005) and in immunosuppressed mice (Ibrahim-Granet et al., 2008). These results imply that propionyl-CoA-generating nutrients are consumed during pathogenesis. Furthermore, accumulation of propionylCoA was shown to inhibit the formation of polyketidederived secondary metabolites such as sterigmatocystin, naphthopyrone, ascoquinone $\mathrm{A}$ and 1,8-dihydroxynaphthalene (DHN) melanin (Maerker et al., 2005; Zhang et al., 2004; Zhang \& Keller, 2004), which seems to be mainly due to an imbalance between the intracellular ratio of propionyl-CoA and acetyl-CoA.

Plant-pathogenic fungi, such as various Fusarium species, contain genes coding for putative methylcitrate cycle enzymes in their genomes; however, experimental evidence of a functional methylcitrate cycle in these species has not been provided. Since it is possible that propionyl-CoAgenerating carbon sources such as odd chain fatty acids or amino acids are consumed during plant infection, this pathway might play an important role during pathogenesis and therefore constitute a novel antifungal target. In addition, because several Fusarium species are known to produce highly toxic secondary metabolites during plant infection and colonization of stored foodstuffs (De Lucca, 2007; Jestoi, 2008; Schollenberger et al., 2007; Völkl et al., 2004), inhibition of the methylcitrate pathway could reduce the production of these toxins. For example, propionate, the direct precursor of propionyl-CoA, is frequently added to stored seeds, especially corn, to prevent mould growth and mycotoxin production (Selwet, 2008). Notably, low amounts of propionate are often well tolerated by fungi and used as a nutrient source, increasing rather than reducing growth rates. Therefore, detoxifying mechanisms do exist that allow growth in the presence of propionate.

Last but not least, several Fusarium species are able to cause severe invasive fusariosis in immunocompromised patients, with attributable mortality rates of up to $100 \%$. The Fusarium solani species complex (teleomorph Nectria haematococca) is isolated from approximately two-thirds of patients with fusarial mycoses (Zhang et al., 2006) and seems to be the most virulent Fusarium species complex (Mayayo et al., 1999). This suggests that this species complex is able to readily adapt to growth within infected human tissues. In contrast, Fusarium verticillioides (teleomorph Gibberella moniliformis) is much less frequently isolated from patients, but is a major pathogen of maize and sorghum and can either grow as an endophyte or cause visible disease on the ears, stalks, roots and seedlings
(Brown et al., 2008; Jurgenson et al., 2002). Furthermore, $F$. verticillioides is a major producer of fumonisins, mycotoxins that have a strong carcinogenic potential (Gelderblom et al., 1988). In contrast to Fusarium solani, which is frequently found as a soil saprophyte (Zhang et al., 2006), a major natural reservoir of $F$. verticillioides is within maize seeds, which are colonized by vertical transmission during asymptomatic endophytic growth within plants (Bacon et al., 2001). These lifestyle differences suggest that these two Fusarium species may possess different mechanisms for adapting to changing environmental conditions and growth under nutrient-limited conditions.

To investigate the ability of Fusarium species to cope with the formation of the toxic compound propionyl-CoA, we analysed the methylcitrate synthases from both $F$. solani and $F$. verticillioides and determined the methylcitrate cycle activity from cells grown in vitro under various conditions. Methylcitrate synthases from both species showed a high degree of sequence identity, but biochemical characteristics and activity induction differed between the species, which may influence their ability to utilize and detoxify propionyl-CoA.

\section{METHODS}

Chemicals and growth media. Unless stated otherwise, all media and chemicals used in this study were obtained from Sigma-Aldrich Chemie.

Media and culture conditions for $\boldsymbol{F}$. solani and $\boldsymbol{F}$. verticillioides. In all experiments the isolates F. solani IP 2330.95 (Hue et al., 1999 ) and F. verticillioides IP 2333.95, kindly provided by the Institut Pasteur culture collection (Paris, France), were used. Cultures were routinely incubated at $28-30{ }^{\circ} \mathrm{C}$ and propagated either on solid agar plates or in liquid cultures, which were shaken at 220 r.p.m. on a rotary shaker. For complex media, potato dextrose broth, potato broth, malt extract or Sabouraud medium were used. Defined minimal media, based on Aspergillus minimal medium $\mathrm{pH}$ 6.5, were supplemented with Casamino acids (MP Biomedicals), peptone (AppliChem), glucose, acetate, ethanol or propionate (all in a range between 10 and $100 \mathrm{mM}$ or $1 \%$ ). Media were inoculated with microconidia derived from cultivation of mycelia in peptonecontaining liquid medium (24-48 h). Microconidia were harvested by filtration through Miracloth with subsequent centrifugation at $4000 \mathrm{~g}$ for $10 \mathrm{~min}$. Conidia were washed twice with PBS and stored as spore suspensions for up to 7 days at $4{ }^{\circ} \mathrm{C}$. To harvest mycelia, cultures were monitored microscopically for excessive microconidia formation. Mycelium was retained during filtration over Miracloth, pressed dry and shock frozen in liquid nitrogen until further use. Cellfree extracts were prepared by grinding the mycelia in the presence of liquid nitrogen, resuspension in $50 \mathrm{mM}$ HEPES buffer $\mathrm{pH} 7.5$ and removal of cell debris by 5 min centrifugation at $16000 \mathrm{~g}$.

Identification of methylcitrate synthase coding sequences. The protein sequence of the $A$. nidulans methylcitrate synthase (accession no. CAB53336) was used as a template for a TBLASTN search against the genome of F. solani (Nectria haematococca; http://genome.jgipsf.org/cgi-bin/runAlignment? $\mathrm{db}=$ Necha2\&advanced=1). A predicted protein with $70 \%$ sequence identity was identified on chromosome 5 and the coding sequence was used for a BLAST search against the genome of $F$. verticillioides (http://www.broadinstitute.org/annotation/genome/fusarium_verticillioides/Blast.html). A hit with high 
identity was found at locus tag FVEG_00189.3. Genome sequences for both Fusarium enzymes were used for ORF prediction by the program GENSCAN (http://genes.mit.edu/GENSCAN.html). To experimentally confirm the open reading frames for both methylcitrate synthases, RNA was isolated from peptone-grown mycelia using the NucleoSpin RNA L kit (Macherey-Nagel) with subsequent Baseline-ZERO DNase treatment (Biozym Scientific). RNA was reverse transcribed into cDNA using anchored Oligo $(\mathrm{dT})_{20}$ primers and reverse transcriptase SuperScript III (Invitrogen). RNA was removed by treatment with $\mathrm{NaOH}$ at $70{ }^{\circ} \mathrm{C}$ and $\mathrm{cDNA}$ was purified by ethanol precipitation in the presence of glycogen. The cDNA was used as template for gene amplification using the oligonucleotides FvMcsASequ_upfo and FvMcsASequ_dore for the F. verticillioides and FsMcsASequ_upfo and FsMcsASequ_dore for the F. solani methylcitrate synthases (Table 1). Gene-specific cDNAs were cloned into the pJET1.2 (Fermentas) cloning vector and sequenced from both strands.

Cloning of methylcitrate synthase genes for recombinant overproduction in $\boldsymbol{E}$. coli. Analysis of the ORF for both methylcitrate synthases revealed a mitochondrial import sequence (http://ihg2.helmholtz-muenchen.de/ihg/mitoprot.html), which was removed before heterologous enzyme production in $E$. coli. The cDNAs from the respective species were used for amplification of the genes with a proofreading polymerase (Accyzyme; Bioline) using oligonucleotides FsMcsABam_for and FsMcsAHind_rev for the $F$. solani enzyme and FvMcsABam_for and FvMcsANot_rev for the $F$. verticillioides enzyme. The PCR products were cloned into the pJET1.2blunt vector (Fermentas) and sequenced from both strands. Internal BamHI sites in both the F. solani and the F. verticillioides coding sequences prohibited direct subcloning into the expression vector. Therefore, the internal BamHI sites were mutated using the FlipFlop Site-Directed Mutagenesis kit as recommended by the manufacturer (Bioline) and the oligonucleotides FsMutBamMcs_for and FsMutBamMcs_rev for F. solani mcsA and FvMcsMutBam_for and FvMcsMutBam_rev for F. verticillioides mcsA. Amplified vectors were transferred into XL1-Blue Supercompetent cells (Stratagene Europe) and the sequences of the mutated genes were confirmed by $B a m H I$ restriction and sequencing. The genes were excised from the pJET1.2blunt vector with BamHI and HindIII for the F. solani enzyme and with BamHI and NotI for the F. verticillioides enzyme and subsequently ligated into a modified pET43.1a vector (Novagen/
Merck) containing an N-terminal His-tag and a TEV protease cleavage site (Hortschansky et al., 2007). Plasmids were then transferred into E. coli BL21(DE3) Rosetta 2 cells (Novagen/Merck), which were used for overproduction of the recombinant enzymes.

Heterologous methylcitrate synthase production and enzyme purification. F. solani and F. verticillioides methylcitrate synthases were purified from E. coli BL21(DE3) Rosetta 2 cells harbouring the appropriate pET43.1 expression vectors. Cells were grown for $26 \mathrm{~h}$ at $28{ }^{\circ} \mathrm{C}$ in $15 \mathrm{ml}$ Overnight express instant TB medium (Novagen/ Merck) in $50 \mathrm{ml}$ flasks on a rotary shaker at 220 r.p.m. Cells were harvested by centrifugation at $4000 \mathrm{~g}$ and cell pellets were resuspended in $3 \mathrm{ml}$ of $50 \mathrm{mM}$ HEPES buffer $\mathrm{pH} 7.5$ (buffer A). Cells were sonicated on ice for $3 \times 2 \mathrm{~min}$ at $70 \%$ intensity with a pulse rate of $50 \%$ and a sonotrode with a diameter of $3 \mathrm{~mm}$ (Sonoplus homogenizer; Bandelin Electronic). Debris was removed by centrifugation at $16800 \mathrm{~g}$ and the supernatant was loaded on a gravity-flow Ni-NTA agarose column (bed volume $1 \mathrm{ml}$; Qiagen) previously equilibrated with buffer A. The column was washed with 8 column volumes of buffer A containing $30 \mathrm{mM}$ imidazole and methylcitrate synthase was eluted in buffer A with $200 \mathrm{mM}$ imidazole. Purity of fractions was confirmed by SDS-PAGE analysis on NuPage Bistris $4-12 \%$ gradient gels in a MES-buffered running system (Invitrogen). Pure fractions were combined and desalted against $40 \mathrm{mM}$ potassium phosphate buffer $\mathrm{pH} 7.0$ using centrifugal filter devices with a cut-off of $30 \mathrm{kDa}$ (Millipore). Glycerol was added to a final concentration of $50 \%(\mathrm{v} / \mathrm{v})$ and samples were stored at $-20{ }^{\circ} \mathrm{C}$.

Methylcitrate synthase assay and biochemical characterization of methylcitrate synthases. The standard assay for methylcitrate synthase (citrate synthase) activity is based on the reduction of 5,5'-dithiobis-3-nitrobenzoate by $\mathrm{CoASH}$ released during the condensation of propionyl-CoA (acetyl-CoA) and oxaloacetate. Assays were performed as described previously (Brock et al., 2000). Temperature stability was tested by incubation of the enzyme for different times at various temperatures followed by an activity determination under standard conditions. Temperature dependency was analysed by preheating the assay mixture to the desired temperature using a water bath connected to the UV/Vis doublebeam spectrophometer that was used in all enzymic analyses (Lambda 25 UV/VIS, Perkin Elmer). The effect of $\mathrm{pH}$ on activity was

Table 1. Oligonucleotides used in this study

Sequences of restriction sites are shown in bold letters. Bases altered for mutation of a restriction site are shown in bold italic.

\begin{tabular}{|c|c|c|}
\hline Name & Sequence $5^{\prime} \rightarrow 3^{\prime}$ & Function \\
\hline Anchored Oligo $(\mathrm{dT})_{20}$ & NVT TTT TTT TTT TTT TTT TTT T & cDNA synthesis \\
\hline \multicolumn{3}{|c|}{ Fusarium solani } \\
\hline FsMcsASequ_upfo & CCA АТT САТ СТС СТС ТСA САC & Amplification $m c s A$ \\
\hline FsMcsASequ_dore & GAC ATG TCC CAA ATT CCA ATC G & Amplification $m c s A$ \\
\hline FsMcsABam_for & GGA TCC ACG GCT GAG CCC GAC & Overexpression $m c s A$ \\
\hline FsMcsAHind_rev & AAG CTT ACT GGC CCT CAA CCT GC & Overexpression $m c s A$ \\
\hline FsMutBamMcs_for & GAT GGA TTC TCC AGA TGA AG & Mutation BamHI site \\
\hline FsMutBamMcs_rev & CTT CAT CTG GAG AAT CCA TC & Mutation BamHI site \\
\hline \multicolumn{3}{|c|}{ Fusarium verticillioides } \\
\hline FvMcsASequ_upfo & CTC CTC ATA CTT GAT CTC TTC & Amplification $m c s A$ \\
\hline FvMcsASequ_dore & CTC TGT CAC GAC GCT ATA TAC & Amplification $m c s A$ \\
\hline FvMcsABam_for & GGA TCC GCA GAG CCT GAT CTC AAG & Overexpression $m c s A$ \\
\hline FvMcsANot_rev & GCG GCC GCT TAT TGA CCC TCA ACC TGC & Overexpression $m c s A$ \\
\hline FvMcsMutBam_for & GTG CTC TGT CTG ATC CTT TCC & Mutation BamHI site \\
\hline FvMcsMutBam_rev & GGA AAG GAT CAG ACA GAG CAC & Mutation BamHI site \\
\hline
\end{tabular}


determined by replacing the standard Tris/ $\mathrm{HCl}$ assay buffer $\mathrm{pH} 8.0$ with a buffer system containing a mixture of boric acid, acetic acid and phosphoric acid (all $0.1 \mathrm{M}$ ) adjusted with $\mathrm{NaOH}$ to the desired $\mathrm{pH}$ values $\left(500 \mu \mathrm{l}\right.$ buffer per $1 \mathrm{ml}$ assay). $K_{\mathrm{m}}$ values for propionylCoA, acetyl-CoA and oxaloacetate were determined using the standard assay in which the concentration of one substrate was held constant whereas that of the other was varied. $K_{\mathrm{m}}$ values were calculated from double reciprocal Lineweaver-Burk plots.

Purification of methylcitrate from $A$. nidulans $\Delta m c s A$ culture broth and determination of methylcitrate cycle and isocitrate lyase activity. Methylcitrate cycle activity was determined by following the conversion of methylcitrate to pyruvate and subsequent reduction to lactate. To obtain pure $2 S, 3 S-2$-methylcitrate, an $A$. nidulans methylcitrate dehydratase deletion strain $\Delta m c d A$ (unpublished) was grown for $72 \mathrm{~h}$ on a medium containing $50 \mathrm{mM}$ acetate and $20 \mathrm{mM}$ propionate. The $\Delta m c d A$ strain is unable to convert methylcitrate to methylaconitate, thus excreting methylcitrate into the growth medium. After removing the mycelium by filtration, a $2 \mathrm{M}$ $\mathrm{BaCl}_{2}$ solution was added to the medium until no further precipitation occurred. The precipitate was collected, dried and mixed with Dowex W $50 \times 8\left(\mathrm{H}^{+}\right.$-form; Fluka). The supernatant contained free methylcitric acid, which was subsequently concentrated on a rotary evaporator, from which a yellowish oil was recovered. GC-MS analysis revealed no major contaminants in the methylcitric acid preparation. A $20 \mathrm{mM}$ methylcitrate stock solution was prepared and used as substrate for methylcitrate cycle activity determination. The assay, performed in a final volume of $1 \mathrm{ml}$, contained the following: $0.2 \mathrm{mM}$ methylcitric acid, $2 \mathrm{mM}$ dithiothreitol, $5 \mathrm{mM} \mathrm{MgCl}_{2}$, 2 U lactate dehydrogenase from rabbit muscle (Fluka), $0.2 \mathrm{mM} \mathrm{NADH}$, different amounts of cell-free extract and $50 \mathrm{mM}$ HEPES buffer $\mathrm{pH}$ 7.5. The reaction was started by the addition of either methylcitric acid or cell-free extract and the decline in absorbance at $340 \mathrm{~nm}$ was monitored over a period of $15 \mathrm{~min}$. Specific activity was defined as the oxidation of $1 \mu \mathrm{mol} \mathrm{NADH} \mathrm{min}^{-1}$ $(\mathrm{mg} \text { protein })^{-1}$ using a millimolar absorption coefficient of $6.2 \mathrm{mM}^{-1} \mathrm{~cm}^{-1}$.

Isocitrate lyase activity was determined based on the formation of glyoxylate-phenylhydrazone from glyoxylate and phenylhydrazine. The assay was performed as previously described (Ebel et al., 2006) and a millimolar absorption coefficient of $16.8 \mathrm{mM}^{-1} \mathrm{~cm}^{-1}$ at $324 \mathrm{~nm}$ was used for calculation of enzyme activity.

Data reproducibility. All growth experiments on solid media were performed in biological triplicates and representative photographs are shown. For determination of enzymic activities after growth on different carbon sources, two independent cell-free extracts were prepared from each condition. From each extract three activity determinations were perfomed. Tables represent mean values and their standard deviation.

\section{RESULTS}

\section{Growth of $\boldsymbol{F}$. solani and $\boldsymbol{F}$. verticillioides on different carbon sources}

To test the general ability of F. solani (strain IP 2330.95) and $F$. verticillioides (strain IP 2333.95) to grow on defined media, we point-inoculated agar plates, containing different carbon sources, with $1 \times 10^{4}$ conidia from fresh microconidia suspensions and incubated plates at $30{ }^{\circ} \mathrm{C}$ for 3 days (Fig. 1). Both strains grew well on the complete media Sabouraud dextrose and malt extract agar. Furthermore, both strains were able to grow on partially or completely hydrolysed proteins (peptone and Casamino acids, respectively). Interestingly, on serum albumin, a potential nutrient source during infection of a human host, rapid germination of $F$. solani, but not $F$. verticillioides, was observed. On all other tested media, growth of $F$. solani was much more pronounced than that of $F$. verticillioides. On glucose, both strains showed some biomass formation, whereas on propionate (propionyl-CoA forming) and also on acetate and ethanol, biomass formation was only observed for F. solani. Furthermore, growth of F. solani was more pronounced on these gluconeogenic carbon sources than on glucose. This observation fits with the saprophytic lifestyle of $F$. solani and suggests that the degradation of carboxylic acids such as acetate and propionate provides nutrient sources during growth in the soil. In contrast, F. verticillioides, which is mainly associated with plants, seems to prefer rich media containing glucose and peptides/amino acids, whereby the latter would also lead to the formation of toxic propionyl-CoA. In order to test whether the rapid adaptation of $F$. solani to

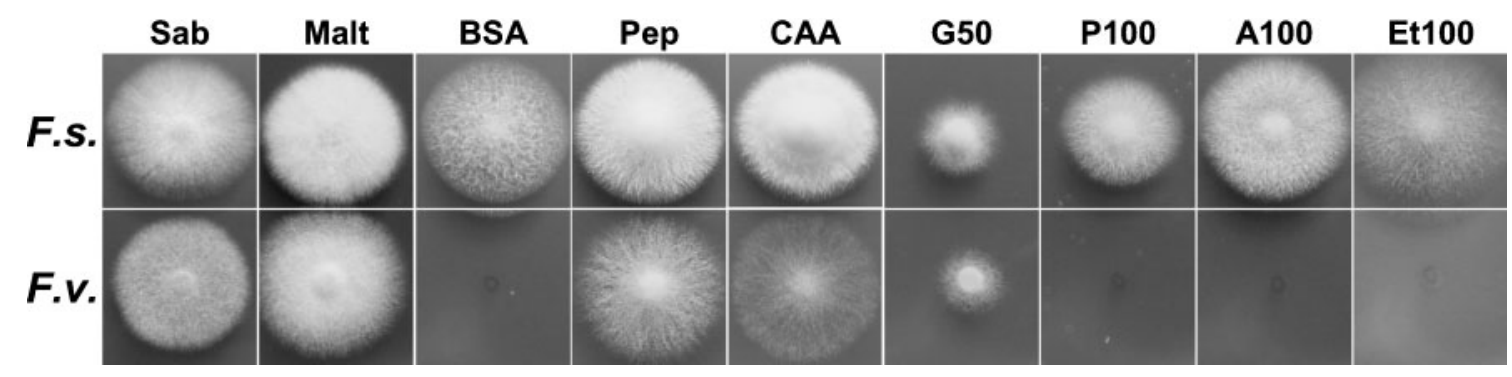

Fig. 1. Growth analysis of F. solani (F.s.) and F. verticillioides (F.v.) on different carbon sources. Plates were inoculated with 10000 microconidia per spot, incubated for 3 days at $30{ }^{\circ} \mathrm{C}$ and photographed. Sab, Sabouraud dextrose medium; Malt, malt extract agar; BSA, $1 \%(\mathrm{w} / \mathrm{v})$ BSA; Pep, $1 \%$ (w/v) peptone; CAA, $1 \%$ (w/v) Casamino acids; G50, $50 \mathrm{mM}$ glucose; P100, $100 \mathrm{mM}$ propionate; A100, $100 \mathrm{mM}$ acetate; $\mathrm{Et} 100,100 \mathrm{mM}$ ethanol. $F$. solani forms colonies under all conditions, whereas $F$. verticillioides only grows in the presence of glucose and/or hydrolysed proteins. No colony formation by F. verticillioides is observed on BSA after the 3 days incubation, indicating a low proteolytic activity. 
propionate is due to a more efficient methylcitrate synthase, which would remove even low amounts of toxic propionyl-CoA, we sought to identify and characterize the respective enzymes from both species.

\section{Identification of methylcitrate synthase coding regions}

The genomes of F. solani (teleomorph Nectria haematococca) and F. verticillioides (teleomorph Gibberella moniliformis) have recently been sequenced and their genome annotation is in progress. To date, only the coding sequences of the methylcitrate synthases ( $m c s A$; EC 2.3.3.5) from the filamentous fungi $A$. nidulans (Brock et al., 2000) and A. fumigatus (Maerker et al., 2005) have been experimentally verified and, due to their high sequence identity to the respective citrate synthases accompanied by a mitochondrial localization signal for both methylcitrate and citrate synthases, an unambigious annotation for other fungal species is rather difficult. Nevertheless, we expected that among filamentous fungi the $\operatorname{mcs} A$ sequences should display higher conservation than those coding for citrate synthases. Therefore, we performed a translated BLAST search against the genome of $F$. solani using the protein sequence of the A. nidulans McsA as a query. The search identified a sequence (ID 69757 ) of 472 amino acids that displayed $70 \%$ identity to the $A$. nidulans McsA query. In addition, the protein showed a sequence identity of $96 \%$ to a conserved hypothetical protein from the close relative Fusarium graminearum (Gibberella zeae; accession no. XP_380351).

The same search against the $F$. verticillioides genome revealed a predicted protein (locus tag FVEG_00189.3) with $71 \%$ identity to the $A$. nidulans McsA. However, the F. verticillioides protein consisted of only 393 amino acids. A BLAST search of the NCBI database (http://blast. ncbi.nlm.nih.gov/Blast.cgi) implied that the predicted protein sequence contained $\mathrm{N}$-terminal and C-terminal truncations. Therefore, the genomic sequences surrounding locus tag FVEG_00189.3 were downloaded and manually checked for the missing coding sequences using the gene prediction program GENSCAN.

To experimentally confirm the open reading frames from both species, oligonucleotides located approximately 50 nucleotides upstream and downstream of the 'self-predicted' start and stop codons were designed. After PCR amplification from total cDNA and subcloning of the PCR products, two independent clones from each species were sequenced. Interestingly, the cDNA sequence of $F$. solani IP 2330.95 (accession no. FN400886) showed a total of 38 nucleotide exchanges (mainly in the wobble position three of the codons) when compared to the published sequence of strain F. solani MPVI 77-13-4. Only two nucleotide exchanges (codon position 2 affected) led to a modification of the deduced amino acid sequence (at amino acid 138, glycine to valine, and position 390, arginine to glutamine). However, due to the phylogenetic diversity of the F. solani species complex, which includes more than 45 phylogenetic and/or biological species (O'Donnell et al., 2008; Zhang et al., 2006), we expected such minor differences in the coding region of the methylcitrate synthase gene when compared to the sequenced strain.

Sequencing of the F. verticillioides cDNA (accession no. FN400887) confirmed the manually deduced amino acid sequence and revealed a protein of 472 amino acids, the same size as determined for the $F$. solani enzyme. Only five nucleotides of the cDNA sequence did not match with those of the sequenced strain F. verticillioides 7600 . These differences only occurred in wobble positions and did not alter the final amino acid sequence. Like the previously studied Aspergillus enzymes, the methylcitrate synthases from F. solani and F. verticillioides contained a mitochondrial import sequence with a cleavage site at amino acid position 30, as predicted by the programs MITOPROT and PSORT (www.expasy.ch). Removal of the 29 amino acid leader peptide leads to mature enzymes containing 443 amino acids and with molecular masses of $49 \mathrm{kDa}$. Comparison of the genomic sequences of the methylcitrate synthases revealed two introns at similar positions. The first intron is located within the mitochondrial import sequence at the same position as the first introns of the methylcitrate synthases of $A$. nidulans and A. fumigatus (accession no. CAI61947). The second intron is located within the coding sequence. Both introns from both Fusarium species follow the GT-AG rule for intron splicing (Breathnach et al., 1978).

\section{Heterologous production of recombinant methylcitrate synthases}

For heterologous overproduction of the Fusarium methylcitrate synthases in E. coli we subcloned the respective cDNAs without the mitochondrial import sequence to yield correctly processed mature enzymes. To facilitate cloning into the modified pET43.1 expression vector, which contains an $\mathrm{N}$-terminal His-tag and a TEV protease cleavage site (Hortschansky et al., 2007), we removed the internal BamHI restriction site from both genes by mutation without altering the encoded amino acids. This strategy enabled the cloning of the $F$. solani enzyme using BamHI and HindIII restriction sites, whereas the $F$. verticillioides enzyme was subcloned using BamHI and NotI restriction sites. Overproduction was performed at $26{ }^{\circ} \mathrm{C}$ in Overnight express instant TB medium and crude extract measurements showed high methylcitrate synthase activity of $3.0 \mathrm{U} \mathrm{mg}^{-1}$ for the $F$. solani and $2.0 \mathrm{U} \mathrm{mg}^{-1}$ for the $F$. verticillioides overproduction approach. The background activity in E. coli cells harbouring an empty pET43.1a vector was $<0.1 \mathrm{U} \mathrm{mg}^{-1}$. When cells were grown at temperatures above $28{ }^{\circ} \mathrm{C}$, significant proportions of the enzymes accumulated within insoluble inclusion bodies. The enzymes were purified from cell-free extracts by $\mathrm{Ni}$ chelate chromatography and the eluted fractions analysed for enzyme purity by SDS-PAGE analysis (Fig. 2). Both 
A

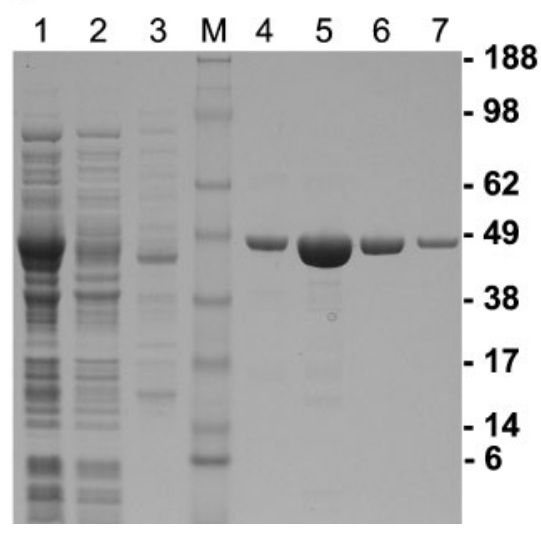

B

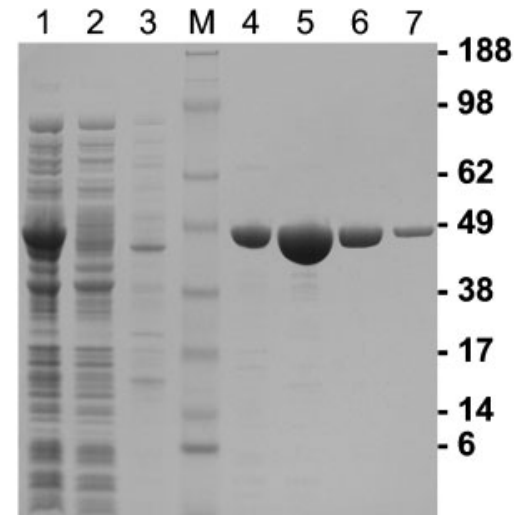

Fig. 2. SDS gel analysis of purified recombinant methylcitrate synthases from $F$. solani (A) and F. verticillioides (B) heterologously produced in E. coli. For both purifications the gels were loaded using the same scheme. Lanes: 1, cell-free extract; 2, flowthrough; 3, wash fraction; 4-7, eluted fractions $1-4 ; M$, molecular mass marker (SeeBlue Plus2 Prestained mass marker; Invitrogen). The purified proteins show a molecular mass of approximately $49 \mathrm{kDa}$, which fits well with the predicted mass of a single subunit of the methylcitrate synthases. enzymes were purified to approximately $99 \%$ homogeneity and showed the expected subunit molecular mass of approximately $49 \mathrm{kDa}$. The specific activity of the purified methylcitrate synthase from $F$. solani (FsMcsA) was $19.55 \mathrm{U} \mathrm{mg}^{-1}$, whereas that of the $F$. verticillioides enzyme (FvMcsA) was $15.31 \mathrm{U} \mathrm{mg}^{-1}$. Treatment with TEV protease (kindly provided by P. Hortschansky, HKI Jena, Germany) for the removal of the N-terminal His-tag did not alter the specific activity of either enzyme (data not shown) and was therefore omitted for further biochemical characterizations. To verify that the purified enzymes originated from the respective cDNAs, protein spots were excised from the gels and subjected to tryptic digestion followed by peptide mass analysis and peptide sequencing by MS/MS analysis. For FsMcsA, 13 peptides exactly matched the predicted sequence, with a total sequence coverage of $33.4 \%$. The sequencing of two peptides by MS/ MS analysis additionally confirmed the origin of the purified enzyme. For FvMcsA, peptide analyses showed a sequence coverage of $31.7 \%$ with 11 peptides matching to the predicted sequence, and sequencing of two peptides also confirmed the origin of the protein.

\section{Biochemical characterization of methylcitrate synthases}

Methylcitrate synthases from aspergilli have been shown to be active not only with propionyl-CoA but also with acetylCoA, thereby acting as both methylcitrate and citrate synthases. Determination of maximum activities showed that citrate synthase activity exceeded that of methylcitrate synthase activity in Aspergillus, but the $K_{\mathrm{m}}$ value for propionyl-CoA was always lower than that for acetyl-CoA, leading to similar catalytic efficiencies for both enzymic activities (Brock et al., 2000; Maerker et al., 2005). We therefore determined both the methylcitrate and citrate synthase biochemical parameters of the Fusarium enzymes (Table 2). Interestingly, the Fusarium methylcitrate synthases differed in their biochemical parameters, despite sharing $96 \%$ amino acid identity. For FsMcsA, citrate

Table 2. Comparison of the biochemical parameters of the methylcitrate synthases from $F$. solani and $F$. verticillioides

\begin{tabular}{|lcc|}
\hline Parameter & McsA F. solani & McsA F. verticillioides \\
\hline Specific activity (propionyl-CoA) & $19.55 \mathrm{U} \mathrm{mg}^{-1}$ & $15.31 \mathrm{U} \mathrm{mg}^{-1}$ \\
Specific activity (acetyl-CoA) & $19.05 \mathrm{U} \mathrm{mg}^{-1}$ & $36.6 \mathrm{U} \mathrm{mg}^{-1}$ \\
Turnover number (propionyl-CoA) & $15.9 \mathrm{~s}^{-1}$ & $12.5 \mathrm{~s}^{-1}$ \\
Turnover number (acetyl-CoA) & $15.5 \mathrm{~s}^{-1}$ & $29.9 \mathrm{~s}^{-1}$ \\
$K_{\mathrm{m}}$ value propionyl-CoA & $1.94 \mu \mathrm{M}$ & $1.88 \mu \mathrm{M}$ \\
$K_{\mathrm{m}}$ value acetyl-CoA & $1.26 \mu \mathrm{M}$ & $2.71 \mu \mathrm{M}$ \\
$K_{\mathrm{m}}$ value oxaloacetate (propionyl-CoA) & $2.58 \mu \mathrm{M}$ & $2.81 \mu \mathrm{M}$ \\
$K_{\mathrm{m}}$ value oxaloacetate (acetyl-CoA) & $3.85 \mu \mathrm{M}$ & $9.64 \mu \mathrm{M}$ \\
Catalytic efficiency (propionyl-CoA) & $8.2 \times 10^{6} \mathrm{~s}^{-1} \mathrm{M}^{-1}$ & $6.6 \times 10^{6} \mathrm{~s}^{-1} \mathrm{M}^{-1}$ \\
Catalytic efficiency (acetyl-CoA) & $1.2 \times 10^{7} \mathrm{~s}^{-1} \mathrm{M}^{-1}$ & $1.1 \times 10^{7} \mathrm{~s}^{-1} \mathrm{M}^{-1}$ \\
Catalytic efficiency (oxaloacetate with propionyl-CoA) & $6.2 \times 10^{6} \mathrm{~s}^{-1} \mathrm{M}^{-1}$ & $4.4 \times 10^{6} \mathrm{~s}^{-1} \mathrm{M}^{-1}$ \\
Catalytic efficiency (oxaloacetate with acetyl-CoA) & $4.0 \times 10^{6} \mathrm{~s}^{-1} \mathrm{M}^{-1}$ & $3.1 \times 10^{6} \mathrm{~s}^{-1} \mathrm{M}^{-1}$ \\
$V_{\text {max }}$ temperature & $49-54{ }^{\circ} \mathrm{C}$ & $49-54{ }^{\circ} \mathrm{C}$ \\
$V_{\text {max }}$ pH & $8.0-9.0$ & $8.0-9.0$ \\
Temperature stability 50 ${ }^{\circ} \mathrm{C}$ (residual activity) & $50 \%$ after $15 \mathrm{~min}$ & $88 \%$ after $60 \mathrm{~min}^{-1}$ \\
\hline
\end{tabular}


synthase activity was slightly lower than methylcitrate synthase activity, while the $K_{\mathrm{m}}$ value for acetyl-CoA was lower than that for propionyl-CoA, leading to similar catalytic efficiencies for both activities. Due to the low $K_{\mathrm{m}}$ for propionyl-CoA $(<2 \mu \mathrm{M})$ together with the low $K_{\mathrm{m}}$ for oxaloacetate $(2.6 \mu \mathrm{M})$ in the presence of propionyl-CoA, it can be assumed that the enzyme is capable of efficiently removing intracellular propionyl-CoA and thus avoiding toxic accumulation of this metabolite.

In contrast, the FvMcsA displayed a two times higher specific activity for acetyl-CoA than for propionyl-CoA and a lower $K_{\mathrm{m}}$ value for propionyl-CoA than for acetyl-CoA. In addition, the $K_{\mathrm{m}}$ value for oxaloacetate in the presence of propionyl-CoA was approximately 4.3 times lower than that in the presence of acetyl-CoA. This indicates that, despite the higher citrate synthase maximum activity, the enzyme can also function as an efficient methylcitrate synthase under in vivo conditions. Therefore, methylcitrate synthase removes toxic propionyl-CoA and produces methylcitrate for the methylcitrate cycle. In contrast, the citric acid cycle specific citrate synthase catalyses the condensation of acetyl-CoA and oxaloacetate for citrate production. Further differences in the biochemical properties of the two enzymes were noted: although both enzymes displayed maximum activity between 49 and $54{ }^{\circ} \mathrm{C}$ and between $\mathrm{pH} 8.0$ and 9.0, the F. verticillioides enzyme turned out to be much more thermostable than the F. solani enzyme (Table 2). However, both enzymes were stable for at least $2 \mathrm{~h}$ at $30{ }^{\circ} \mathrm{C}$, which is the upper optimum growth temperature for both species.

\section{Determination of methylcitrate synthase, methylcitrate cycle and citrate synthase activity under different growth conditions}

To confirm the in vivo presence of a functional methylcitrate synthase (and of the enzymes of the entire methylcitrate cycle), both Fusarium species were grown on different carbon sources and tested for enzymic activities. Preparation of mycelial crude extracts from cells grown directly on different media was not possible because: (i) F. verticillioides did not grow on ethanol or propionate to biomasses sufficient for crude protein extract; (ii) $F$. verticillioides tended to produce large amounts of microconidia on several media rather than producing mycelium.

To circumvent these problems, we decided to pre-grow both species on media that allowed optimal mycelia production. Growth tests revealed that $F$. solani produced high amounts of mycelia within $24 \mathrm{~h}$ when inoculated into minimal medium containing $100 \mathrm{mM}$ glucose as the sole carbon and energy source. In contrast, the most favourable condition for $F$. verticillioides mycelia production was incubation for $24 \mathrm{~h}$ in Sabouraud dextrose broth. Mycelia were harvested, washed with $0.6 \mathrm{M} \mathrm{KCl}$ solution and aliquots were transferred to media containing different carbon sources (glucose, Casamino acids, ethanol or propionate). The mycelia were incubated in these media for $7 \mathrm{~h}$ at $30{ }^{\circ} \mathrm{C}$ on a rotary shaker. The presence of intact mycelia and the absence of significant microconidia formation was confirmed regularly by microscopy.

To confirm that $7 \mathrm{~h}$ incubation was sufficient for adaptation to the new carbon sources, we tested cell-free extracts of mycelia shifted to ethanol for activity of the glyoxylate cycle marker enzyme isocitrate lyase (Icl). This cycle is essential for the provision of oxaloacetate during growth on ethanol, and high Icl activity denotes an adaptation to the growth medium. The specific Icl activity for $F$. solani increased from $1.0 \pm 0.1 \mathrm{mU} \mathrm{mg}^{-1}$ to $295 \pm 7 \mathrm{mU} \mathrm{mg}^{-1}$ after the $7 \mathrm{~h}$ shift to ethanol. For F. verticillioides the Icl activity increased from $9.7 \pm 1.0 \mathrm{mU} \mathrm{mg}^{-1}$ to $342 \pm 4 \mathrm{mU} \mathrm{mg}^{-1}$. This indicates that both strains produced glyoxylate cycle enzymes for the utilization of ethanol. Therefore, methylcitrate synthase and methylcitrate cycle, as well as citrate synthase activities, were determined for both species under all growth conditions (Table 3).

The overall activity determination of the methylcitrate cycle consisted of the actions of methylcitrate dehydratase, methylisocitrate dehydratase and methylisocitrate lyase and hence represented the activity of the rate-limiting enzyme. Moreover, because only low levels of methylcitrate were present during determination of cycle activity, whilst methylcitrate synthase activity was measured under $V_{\max }$ conditions (substrate saturation), measured cycle activity never exceeded that of synthase activity. Nevertheless, increase of methylcitrate synthase activity was always accompanied by increased methylcitrate cycle activity, implying that the enzymes of the entire pathway follow the same scheme of regulation.

We also determined citrate synthase activity, because methylcitrate synthases have both methylcitrate and citrate synthase activity, whereas the citric acid cycle citrate synthases (characterized so far) are substrate-specific. Purified FsMcsA displayed similar methylcitrate and citrate synthase activity, and citrate synthase activity exceeding a ratio of $1: 1$ (Cs:Mcs) signified production of the citric acid cycle specific citrate synthase. In contrast, the purified FvMcsA displayed a higher citrate synthase maximum activity, and a ratio exceeding 2.4:1 (Cs:Mcs) was indicative of the formation of the specific citrate synthase. In both cases, the smaller the Cs: Mcs ratios, the higher the specific production of methylcitrate synthase activity. As shown in Table 3, the strongest methylcitrate synthase activity was observed on Casamino acids and propionate both propionyl-CoA-generating nutrient sources. Surprisingly, $F$. verticillioides displayed an extremely high citrate synthase activity on Casamino acids. This was unexpected because Casamino acids are also rich in amino acids such as glutamate, which lead to citric acid cycle intermediates and generally suppress citrate synthase activity (as observed for $F$. solani). In addition, $F$. verticillioides displayed the highest specific methylcitrate synthase activity on Casamino acids, whereas F. solani showed strongest activity on propionate. 
Table 3. Methylcitrate synthase, citrate synthase and methylcitrate cycle activity of $F$. solani and $F$. verticillioides grown for $7 \mathrm{~h}$ on different carbon sources

\begin{tabular}{|c|c|c|c|c|c|}
\hline Growth condition & $\begin{array}{l}\text { Methylcitrate cycle } \\
(\mathrm{MC})\left(\mathrm{mU} \mathrm{mg}^{-1}\right)\end{array}$ & 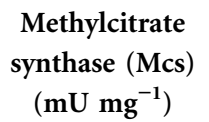 & $\begin{array}{l}\text { Citrate synthase (Cs) } \\
\qquad\left(\mathrm{mU} \mathrm{mg}^{-1}\right) \ddagger\end{array}$ & Ratio Cs: Mcs $\ddagger$ & Ratio MC: Mcs \\
\hline \multicolumn{6}{|l|}{ F. solani ${ }^{\star}$} \\
\hline Glucose $(50 \mathrm{mM})$ & $8 \pm 1.0$ & $21.7 \pm 0.2$ & $274 \pm 5(260)$ & $12.6: 1(12.0: 1)$ & $1: 2.7$ \\
\hline Ethanol $(50 \mathrm{mM})$ & $14.6 \pm 0.2$ & $32.4 \pm 0.2$ & $400 \pm 10(368)$ & $12.3: 1(11.6: 1)$ & $1: 2.2$ \\
\hline Casamino acids (1\%) & $10.0 \pm 0.2$ & $35.6 \pm 2.0$ & $210 \pm 13(175)$ & $5.9: 1(4.9: 1)$ & $1: 3.6$ \\
\hline Propionate $(20 \mathrm{mM})$ & $37.4 \pm 2.0$ & $145 \pm 3$ & $615 \pm 27(474)$ & $4.2: 1(3.3: 1)$ & $1: 3.9$ \\
\hline \multicolumn{6}{|l|}{ F. verticillioides $\dagger$} \\
\hline Glucose $(50 \mathrm{mM})$ & $3.8 \pm 0.5$ & $6.2 \pm 0.6$ & $369 \pm 9(354)$ & $59.5: 1(57.1: 1)$ & $1: 1.6$ \\
\hline Ethanol (50 mM) & $17.1 \pm 1.0$ & $29.5 \pm 1.0$ & $681 \pm 13(610)$ & $23.1: 1(20.7: 1)$ & $1: 1.7$ \\
\hline Casamino acids (1\%) & $27.7 \pm 2.5$ & $81.7 \pm 1.5$ & $867 \pm 28(672)$ & $10.6: 1(8.2: 1)$ & $1: 2.9$ \\
\hline Propionate (20 mM) & $38.2 \pm 3.0$ & $69.8 \pm 1.0$ & $683 \pm 24(516)$ & $9.8: 1(7.4: 1)$ & $1: 1.8$ \\
\hline
\end{tabular}

*Mycelia were harvested from $100 \mathrm{mM}$ glucose minimal medium, washed and transferred to fresh medium as indicated.

$\dagger$ Mycelia were harvested from Sabouraud glucose medium, washed and transferred to fresh medium as indicated.

$¥$ Numbers in parentheses denote citrate synthase activity after subtraction of activity deriving from methylcitrate synthases (factor 0.97 F. solani; 2.39 F. verticillioides).

Calculation of the ratios of methylcitrate cycle to methylcitrate synthase activity revealed that F. solani displayed a 3.6- and 3.9-fold higher methylcitrate synthase activity on Casamino acids and propionate, respectively, whereas a similar increase (2.9-fold) for $F$. verticillioides was only observed on Casamino acids and not on propionate. This suggests that, during growth of $F$. verticillioides on propionate, methylcitrate synthase may be the rate-limiting enzyme in the degradation of propionyl-CoA.

\section{DISCUSSION}

In this study we showed that two Fusarium species contain functional methylcitrate synthases and methylcitrate cycle enzymes, which are induced in the presence of propionylCoA-generating carbon sources. Heterologous production of recombinant methylcitrate synthases in E. coli allowed the biochemical characterization of the purified enzymes, revealing that both enzymes, although sharing $96 \%$ identity at the amino acid level, displayed some differences in their biochemical properties. The most striking difference was the balanced methylcitrate and citrate synthase activities of FsMcsA under $V_{\max }$ conditions compared to FvMcsA, which displayed significantly higher citrate synthase activity, as has been described for A. nidulans and A. fumigatus (Maerker et al., 2005). Furthermore, at the substrate-specificity level, the $F$. solani enzyme behaved differently from methylcitrate synthases of Aspergillus species, displaying a lower $K_{\mathrm{m}}$ value for acetyl-CoA than for propionyl-CoA, whereas the $F$. verticillioides enzyme had a higher affinity for propionyl-CoA (Maerker et al., 2005). Despite these differences, both Fusarium enzymes bound oxaloacetate more efficiently in the presence of
propionyl-CoA than in the presence of acetyl-CoA. Such high-efficiency binding of oxaloacetate is a prerequisite for an efficient methylcitrate synthase, because in the cell oxaloacetate may be the rate-limiting factor in the removal of propionyl-CoA. It has been shown for A. niger that the available oxaloacetate concentration for enzymic reactions is below 2-3 $\mu \mathrm{M}$ (Ruijter et al., 2000), indicating that methylcitrate synthases might act near or below their $K_{\mathrm{m}}$ values for this substrate. Taken together, the results indicate that although both enzymes display differences in their substrate affinities for the three substrates (acetylCoA, propionyl-CoA and oxaloacetate), they display very similar methylcitrate synthase catalytic efficiencies. Nevertheless, the different temperature stabilities of the Fusarium enzymes indicate a different stabilization of the protein folding, which seems to be mediated by only a few amino acid exchanges. In this context, it is difficult to predict which of the amino acids are responsible for the observed differences, but further analysis of methylcitrate synthases from other Fusarium species could help answer this question.

A possible candidate gene for comparison derives from $F$. graminearum (Gibberella zeae). The genome annotation of this fungus reveals a putative 'citrate synthase' (protein accession no. XP_380351). This 'citrate synthase' also consists of 472 amino acids with a predicted mitochondrial import sequence encoded by the first 29 amino acids, which is exactly the same as predicted for the methylcitrate synthases from $F$. solani and $F$. verticillioides. A more detailed comparison of the $F$. graminearum enzyme with those of the other two species shows that it displays only eight amino acid differences from the $F$. verticillioides enzyme (four of these located within the mitochondrial import sequence), but 18 differences from the F. solani 
enzyme (one difference located within the mitochondrial import sequence). Therefore, we predict that the $F$. graminearum enzyme also acts as a methylcitrate synthase and its biochemical parameters likely reflect those of the $F$. verticillioides enzyme.

In addition to the biochemical characterization of the recombinant methylcitrate synthases, which showed that functional methylcitrate synthases are indeed present within the genomes of both Fusarium species, we also determined whether functional enzymes of the entire methylcitrate cycle are in fact produced in the presence of propionyl-CoA-generating carbon sources. Our results showed that F. solani displayed the strongest formation of methylcitrate synthase and also the highest cycle activity when cells were grown in the presence of propionate. This result is in agreement with the rapid germination and growth of $F$. solani on propionate-containing medium (Fig. 1). However, although methylcitrate synthase and cycle activity were also present when $F$. solani was grown on Casamino acids, the increase in activity was rather weak, similar to that on ethanol, and only slightly higher than on glucose. This suggests that the F. solani methylcitrate cycle has quite a high basal activity and that this level is sufficient to remove propionyl-CoA derived from amino acid degradation. Another possible explanation is the preferred metabolism of amino acids other than isoleucine, valine and methionine. This would diminish the formation of propionyl-CoA, thus reducing the need for high methylcitrate cycle activity.

In $F$. verticillioides, activity of the methylcitrate synthase and of the entire cycle behaved somewhat differently. On glucose, it appears that carbon catabolite repression occurs, as hardly any methylcitrate synthase and cycle activity was detectable on this carbon source. Slightly higher activity was observed on ethanol, accounting for a release of repression without induction. Interestingly, the highest methylcitrate synthase activity for $F$. verticillioides was observed not on propionate, but on Casamino acids, accompanied by an extremely high citrate synthase activity. Such high citrate synthase activity was unexpected because Casamino acids contain a significant amount of glutamate (14.2\% of all amino acids), which generally represses citrate synthase induction (Burand et al., 1975). Furthermore, citrate synthase activity from $F$. verticillioides was always higher than that from $F$. solani, which accounted for the increased ratio of citrate synthase to methylcitrate synthase activity under all tested growth conditions (Table 3). However, methylcitrate synthase activity was strongly present on Casamino acids and was even higher than that observed on propionate. The differential enhancement of methylcitrate synthase activity by $F$. verticillioides was reflected in the growth of this strain, as F. verticillioides IP 2333.95 hardly grew on propionate but grew well on Casamino acids. Therefore, F. verticillioides would appear to use its methylcitrate cycle to remove propionyl-CoA derived from amino acids such as methionine, isoleucine and valine, which constitute $1.7 \%$,
$2.7 \%$ and $4.1 \%$, respectively, of the total amino acids present in the Casamino acid preparation (http:// www.mpbio.com/product_info.php?products_id=3060-012) used in our experiments. From these analyses we conclude that specific levels of methylcitrate synthase activity reflect the growth behaviour of both Fusarium species.

In summary, we have shown, we believe for the first time, that Fusarium species contain a functional methylcitrate cycle, important for the removal of propionyl-CoA. Interestingly, despite their strong homology, the biochemical properties of these two methylcitrate synthases resulted in quite different phenotypes of the two species during growth on different carbon sources. F. solani, but not $F$. verticillioides, was able to rapidly adapt to and assimilate multiple nutrient sources, perhaps reflecting its saprophytic lifestyle, where, in the soil, it must possess the metabolic flexibility to meet the demands of this environmental niche. F. verticillioides, on the other hand, is in almost constant association with plants and has therefore specialized its metabolic machinery. The high metabolic flexibility of $F$. solani may additionally explain why this species is most frequently isolated from patients with invasive fusariosis. To further analyse the relevance of propionyl-CoA detoxification in plant infection, saprophytic growth and the production of toxic secondary metabolites, deletion of the coding methylcitrate synthase sequences from the genomes of both species will be required. Such future analyses will show whether the methylcitrate cycle and the removal of toxic propionyl$\mathrm{CoA}$ are essential during these processes.

\section{ACKNOWLEDGEMENTS}

We thank G. Steinmetzer for technical assistance during cloning procedures. This work was supported by funds from the Hans-Knoell Institute.

\section{REFERENCES}

Bacon, C. W., Yates, I. E., Hinton, D. M. \& Meredith, F. (2001). Biological control of Fusarium moniliforme in maize. Environ Health Perspect 109 (Suppl. 2), 325-332.

Breathnach, R., Benoist, C., O’Hare, K., Gannon, F. \& Chambon, P. (1978). Ovalbumin gene: evidence for a leader sequence in mRNA and DNA sequences at the exon-intron boundaries. Proc Natl Acad Sci U S A 75, 4853-4857.

Brock, M. (2005). Generation and phenotypic characterization of Aspergillus nidulans methylisocitrate lyase deletion mutants: methylisocitrate inhibits growth and conidiation. Appl Environ Microbiol 71, 5465-5475.

Brock, M. \& Buckel, W. (2004). On the mechanism of action of the antifungal agent propionate. Eur J Biochem 271, 3227-3241.

Brock, M., Fischer, R., Linder, D. \& Buckel, W. (2000). Methylcitrate synthase from Aspergillus nidulans: implications for propionate as an antifungal agent. Mol Microbiol 35, 961-973.

Brock, M., Darley, D., Textor, S. \& Buckel, W. (2001). 2Methylisocitrate lyases from the bacterium Escherichia coli and the 
filamentous fungus Aspergillus nidulans: characterization and comparison of both enzymes. Eur J Biochem 268, 3577-3586.

Brock, M., Maerker, C., Schutz, A., Volker, U. \& Buckel, W. (2002). Oxidation of propionate to pyruvate in Escherichia coli. Involvement of methylcitrate dehydratase and aconitase. Eur J Biochem 269, 61846194.

Brown, D. W., Butchko, R. A. \& Proctor, R. H. (2008). Genomic analysis of Fusarium verticillioides. Food Addit Contam Part A Chem Anal Control Expo Risk Assess 25, 1158-1165.

Burand, J. P., Drillien, R. \& Bhattacharjee, J. K. (1975). Citrate synthaseless glutamic acid auxotroph of Saccharomyces cerevisiae. Mol Gen Genet 139, 303-309.

De Lucca, A. J. (2007). Harmful fungi in both agriculture and medicine. Rev Iberoam Micol 24, 3-13.

Ebel, F., Schwienbacher, M., Beyer, J., Heesemann, J., Brakhage, A. A. \& Brock, M. (2006). Analysis of the regulation, expression, and localisation of the isocitrate lyase from Aspergillus fumigatus, a potential target for antifungal drug development. Fungal Genet Biol 43, 476-489.

Gelderblom, W. C., Marasas, W. F., Jaskiewicz, K., Combrinck, S. \& van Schalkwyk, D. J. (1988). Cancer promoting potential of different strains of Fusarium moniliforme in a short-term cancer initiation/ promotion assay. Carcinogenesis 9, 1405-1409.

Horswill, A. R. \& Escalante-Semerena, J. C. (2001). In vitro conversion of propionate to pyruvate by Salmonella enterica enzymes: 2-methylcitrate dehydratase (PrpD) and aconitase enzymes catalyze the conversion of 2-methylcitrate to 2-methylisocitrate. Biochemistry 40, 4703-4713.

Hortschansky, P., Eisendle, M., Al-Abdallah, Q., Schmidt, A. D., Bergmann, S., Thön, M., Kniemeyer, O., Abt, B., Seeber, B. \& other authors (2007). Interaction of HapX with the CCAAT-binding complex - a novel mechanism of gene regulation by iron. EMBO J 26, 3157-3168.

Hue, F. X., Huerre, M., Rouffault, M. A. \& de Bievre, C. (1999). Specific detection of Fusarium species in blood and tissues by a PCR technique. J Clin Microbiol 37, 2434-2438.

Ibrahim-Granet, O., Dubourdeau, M., Latgé, J. P., Ave, P., Huerre, M., Brakhage, A. A. \& Brock, M. (2008). Methylcitrate synthase from Aspergillus fumigatus is essential for manifestation of invasive aspergillosis. Cell Microbiol 10, 134-148.

Jestoi, M. (2008). Emerging fusarium-mycotoxins fusaproliferin, beauvericin, enniatins, and moniliformin: a review. Crit Rev Food Sci Nutr 48, 21-49.

Jurgenson, J. E., Zeller, K. A. \& Leslie, J. F. (2002). Expanded genetic map of Gibberella moniliformis (Fusarium verticillioides). Appl Environ Microbiol 68, 1972-1979.

Ledley, F. D., Crane, A. M., Klish, K. T. \& May, G. S. (1991). Is there methylmalonyl-CoA mutase in Aspergillus nidulans? Biochem Biophys Res Commun 177, 1076-1081.

Luttik, M. A., Kotter, P., Salomons, F. A., van der Klei, I. J., van Dijken, J. P. \& Pronk, J. T. (2000). The Saccharomyces cerevisiae ICL2 gene encodes a mitochondrial 2-methylisocitrate lyase involved in propionyl-coenzyme A metabolism. J Bacteriol 182, 7007-7013.

Maerker, C., Rohde, M., Brakhage, A. A. \& Brock, M. (2005). Methylcitrate synthase from Aspergillus fumigatus. Propionyl-CoA affects polyketide synthesis, growth and morphology of conidia. FEBS J 272, 3615-3630.

Martens, J. H., Barg, H., Warren, M. J. \& Jahn, D. (2002). Microbial production of vitamin $\mathrm{B}_{12}$. Appl Microbiol Biotechnol 58, 275-285.

Mayayo, E., Pujol, I. \& Guarro, J. (1999). Experimental pathogenicity of four opportunist Fusarium species in a murine model. $J$ Med Microbiol 48, 363-366.

O'Donnell, K., Sutton, D. A., Fothergill, A., McCarthy, D., Rinaldi, M. G., Brandt, M. E., Zhang, N. \& Geiser, D. M. (2008). Molecular phylogenetic diversity, multilocus haplotype nomenclature, and in vitro antifungal resistance within the Fusarium solani species complex. J Clin Microbiol 46, 2477-2490.

Ruijter, G. J., Panneman, H., Xu, D. \& Visser, J. (2000). Properties of Aspergillus niger citrate synthase and effects of citA overexpression on citric acid production. FEMS Microbiol Lett 184, 35-40.

Schollenberger, M., Müller, H. M., Rüfle, M., Terry-Jara, H., Suchy, S., Plank, S. \& Drochner, W. (2007). Natural occurrence of Fusarium toxins in soy food marketed in Germany. Int J Food Microbiol 113, 142-146.

Selwet, M. (2008). Effect of organic acids on numbers of yeasts and mould fungi and aerobic stability in the silage of corn. Pol J Vet Sci 11, 119-123.

Textor, S., Wendisch, V. F., De Graaf, A. A., Müller, U., Linder, M. I., Linder, D. \& Buckel, W. (1997). Propionate oxidation in Escherichia coli: evidence for operation of a methylcitrate cycle in bacteria. Arch Microbiol 168, 428-436.

Uchiyama, H., Ando, M., Toyonaka, Y. \& Tabuchi, T. (1982). Subcellular localization of the methylcitric-acid-cycle enzymes in propionate metabolism of Yarrowia lipolytica. Eur J Biochem 125, 523-527.

Voet, D. \& Voet, J. G. (2004). Biochemistry, 3rd edn. Chichester: Wiley. Völkl, A., Vogler, B., Schollenberger, M. \& Karlovsky, P. (2004). Microbial detoxification of mycotoxin deoxynivalenol. $J$ Basic Microbiol 44, 147-156.

Zhang, Y. Q. \& Keller, N. P. (2004). Blockage of methylcitrate cycle inhibits polyketide production in Aspergillus nidulans. Mol Microbiol 52, 541-550.

Zhang, Y. Q., Brock, M. \& Keller, N. P. (2004). Connection of propionyl-CoA metabolism to polyketide biosynthesis in Aspergillus nidulans. Genetics 168, 785-794.

Zhang, N., O'Donnell, K., Sutton, D. A., Nalim, F. A., Summerbell, R. C., Padhye, A. A. \& Geiser, D. M. (2006). Members of the Fusarium solani species complex that cause infections in both humans and plants are common in the environment. J Clin Microbiol 44, 21862190 .

Edited by: S. D. Harris 